\title{
Filtering and Obtaining Attributes in Seismic Data Using the SSA Method
}

\author{
Leonardo M. Batista *CPGG/UFBA, Milton J. Porsani, CPGG/UFBA
}

Copyright 2019, SBGf - Sociedade Brasileira de Geofísica.

This paper was prepared for presentation at the $16^{\text {th }}$ International Congress of the Brazilian Geophysical Society, held in Rio de Janeiro, Brazil, 19-22 August, 2019.

Contents of this paper were reviewed by the Technical Committee of the $16^{\text {th }}$ International Congress of The Brazilian Geophysical Society and do not necessarily represent any position of the SBGf, its officers or members. Electronic reproduction or storage of any part of this paper for commercial purposes without the written consent of The Brazilian Geophysical Society is prohibited.

\begin{abstract}
With the goal to improve the signal/noise ratio and obtain great information to the reservoir characterization, the decomposition called Singular Spectrum Analysis (SSA) and the other one named as Frequency-timing has shown to be very useful to create images with high quality resolution. To decompose into singular values, it works with a single trace named by Porsani et al. (2017) as filtering with iteractive resource Singular Spectrum Analysis (RI-SSA), which allows to obtain a timing correlation of the events, producing images without the unwelcome noising. Afterwards, the chosen method to decompose was the frequency-timing suggested by Zoukaneri and Porsani (2015) who called it Wigner-Ville Maximum Entropy Method (ME-MAM), which involves the maximum entrophy concept from Burg to get a high resolution power spectrum.
\end{abstract}

\section{Introduction}

Coherent noise attenuation is an important issue in seismic data processing. Several filtering methods has been developed over the years and most of them use a multichannel approach, often leading to poor results due to spatial aliasing. To overcome this, we used the so-called Iteractive Recursive Singular Spectrum Analysis (RI-SSA) introduced by Porsani et al. (2017) to decompose each trace in a shot gather using only the time correlation of the events within it (Silva, 2015). The RI-SSA method was able to separate the signal components more consistently when compared to the well-known Singular Spectrum Analysis (SSA), since the latter mixes the high and low frequency components (Harris and Yuan, 2010).

To generate the seismic attributes (Taner et al., 1994) on the RI-SSA filtered stacked section we use the maximum entropy method (Burg, 1967) to estimate a high resolution spectrum, avoiding the crossed terms. This method, called the Maximum Entropy of Wigner-Ville method (MEMWV) by Zoukaneri and Porsani (2015), is based on the estimation of a linear prediction operator and on the estimation and extension of the autocorrelation function. After calculating the autocorrelation coefficients, one can generate the Kernel terms of the Wigner-Ville Maximum Entropy, which generate the maximum entropy spectrum when applied to the Fourier transform.
Theory

Singular Spectrum Analysis (SSA)

In SSA, we first create a Toeplitz matrix D using the operator $J\{$.$\} , which is applied to the each trace \mathbf{d}=$ $[\mathrm{d}(1), \ldots, \mathrm{d}(\mathrm{M})]^{\top}$, as in eq. 1. D is often called the trajectory matrix (Harris and Yuan, 2010).

$$
J\{\mathbf{d}\}=\mathbf{D}=\left[\begin{array}{cccc}
\mathrm{d}(1) & 0 & \cdots & 0_{N} \\
\vdots & \mathrm{d}(1) & \ddots & \vdots \\
\mathrm{d}(\mathrm{M}) & \vdots & \ddots & 0 \\
0 & \mathrm{~d}(\mathrm{M}) & \vdots & \mathrm{d}(1) \\
\vdots & \vdots & \ddots & \\
0_{M+N-1} & 0 & \cdots & \mathrm{d}(\mathrm{M})
\end{array}\right]
$$

Then we calculate the singular value decomposition of $\mathbf{D}$, given by

$$
\mathbf{D}=\mathbf{U} \Sigma \mathbf{V}^{T}=\sum_{i=1}^{N} \sigma_{i} \mathbf{u}_{i} \mathbf{v}_{i}^{T}=\sum_{i=1}^{N} \widetilde{\mathbf{D}}_{i},
$$

where $\mathbf{U}=\left[\begin{array}{lll}\mathbf{u}_{1} & \ldots & \mathbf{u}_{\mathrm{N}}\end{array}\right]$ is a $(M+N-1) \times(M+N-1)$ matrix containing the left singular vectors, $\mathbf{V}=\left[\mathbf{v}_{1} \ldots \mathbf{v}_{\mathrm{N}}\right]$ is a $N \times N$ matrix with the right singular vectors and $\Sigma=$ $\operatorname{diag}\left(\sigma_{1} \sigma_{2} \ldots \sigma_{\mathrm{N}}\right)$ is a matrix of the singular values, where $\sigma_{1} \geq \sigma_{2} \geq \cdots \geq \sigma_{N} \geq 0$ (Golub and Van Loan, 2012). The matrix $\mathbf{D}$ could be seen as a sum of unitary matrices $\left(u_{i} v_{i}^{T}\right)$ weighted by the respective singular values, named as eigenimages (Freire, 1986).

The seismic trace can be reconstructed applying the inverse operator $J^{-1}\{$.$\} in \widetilde{\mathbf{D}}$. This inverse operator (eq. 3) includes the displacement adjustement and trace average.

$$
\mathbf{d}=\sum_{i=1}^{N} \widetilde{\mathbf{d}}_{i}=\sum_{i=1}^{N} J^{-1}\left\{\widetilde{\mathbf{D}}_{i}\right\}
$$

In order to validate the decomposition, a synthetic trace (Tary et al., 2014) with five components was used. It consists of two harmonic signals of $15 \mathrm{~Hz}$ and $35 \mathrm{~Hz}\left(\mathrm{~s}_{1}\right.$ and $s_{2}$ ), a harmonic signal modulated by frequency around $65 \mathrm{~Hz}\left(\mathrm{~s}_{3}\right)$, a sweep between $15 \mathrm{~Hz}$ and $158 \mathrm{~Hz}\left(\mathrm{~s}_{4}\right)$, and a Morlet wavelet with center frequency $113 \mathrm{~Hz}\left(\mathrm{~s}_{5}\right)$. The related equations can be seen in Figure 1. Note that the trace is the sum of the five components.

Using the algorithm 1, it was possible to decompose the synthetic signal into 15 components (Figure 2), and combine them to generate similar components Figure 3 , in order to restore the components that generated the synthetic trace (Figure 1). 


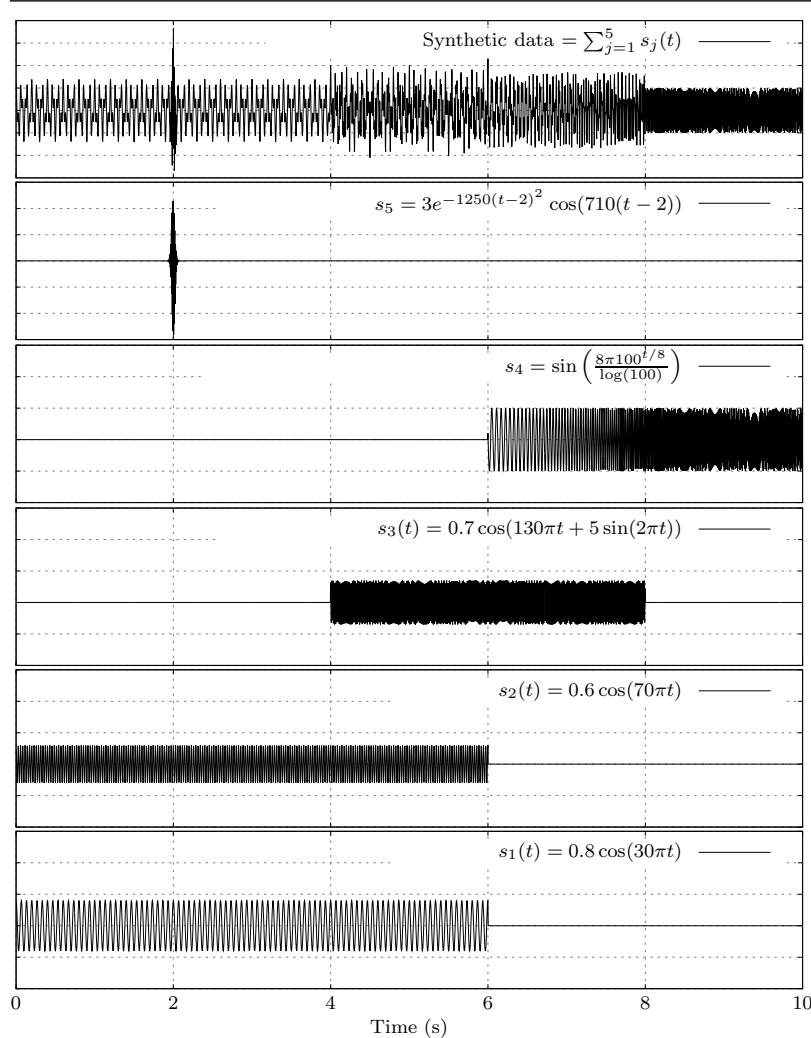

Figure 1: The five components $\mathrm{s}_{1}(\mathrm{t}), \mathrm{s}_{2}(\mathrm{t}), \mathrm{s}_{3}(\mathrm{t}), \mathrm{s}_{4}(\mathrm{t}), \mathrm{s}_{5}(\mathrm{t})$ and the resulting synthetic signal.

\begin{tabular}{l}
\hline Algorithm 1: SSA \\
\hline Generate the matrix of the displaced data, with the $J, \mathbf{D}$ \\
operator \\
Calculate the first eigenvalues and eigenvectors \\
Calculate the first eigenimage \\
Generate the filtered trace using the $J$ inverse operator \\
\hline
\end{tabular}

Recursivo Iterativo Singular Spectrum Analysis (RI-SSA)

Figure 11 shows the result of the SSA; note that it is not possible to properly separate the high and low energies using this technique, leaving a considerable part of the ground roll behind. Thus, to overcome this limitation, Porsani et al. (2018) added to the SSA method the recursion and iteration to properly decode and separate a signal into high and low energy components using three loops. In the internal loop, the high energy component of the signal is calculated by recursion in the number of rows in the trajectory matrix, using only the first eigenvalues and eigenvectors, which correspond to the highest energy component. The result is subtracted from the input signal in the second loop and the process is repeated. This provides an estimate of the low-energy part of the signal. In the external loop, this low energy signal is subtracted from the input signal and the result is output as a signal component. The entire procedure is then repeated with the low energy component as the new input signal. The procedure starts with the search for input data and is repeated until the $K$ signal components have been estimated. Algorithm 2 illustrates this procedute:

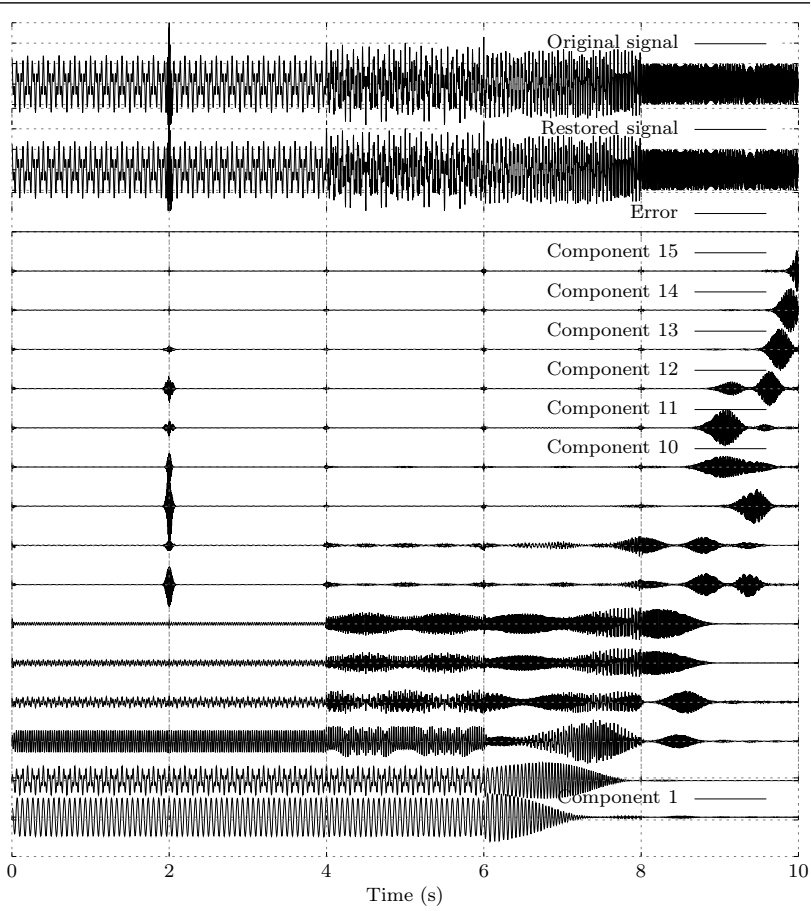

Figure 2: Decomposition of the synthetic signal (Figure 1) using the SSA algorithm

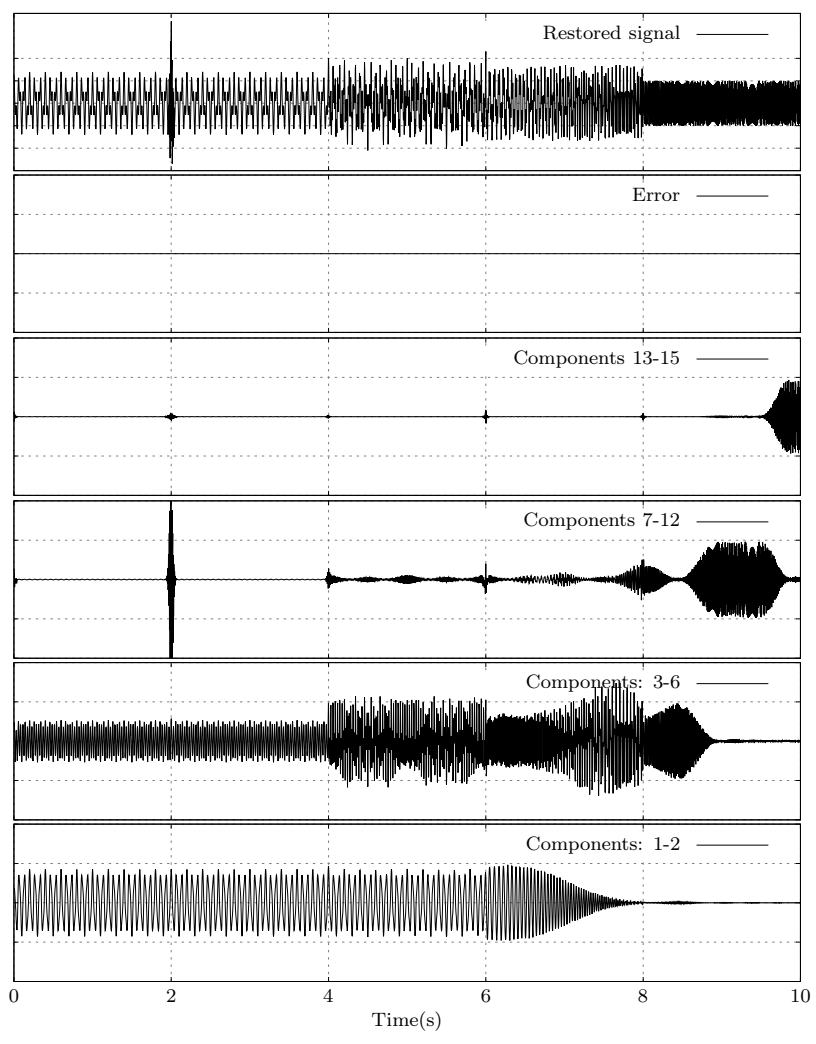

Figure 3: Sum of similar components of Figure 2

Figure 4 shows the result of the synthetic trace decomposition (Figure 1) using the RI-SSA algorithm; note that it was possible to accurately separate the low and high frequency components, which means this method is better 


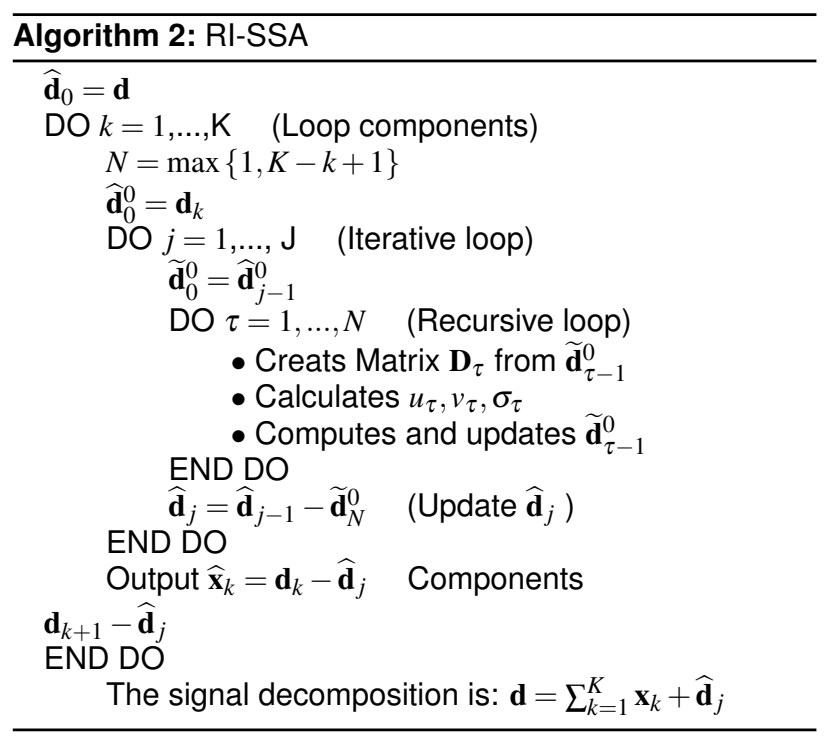

than the SSA 2.

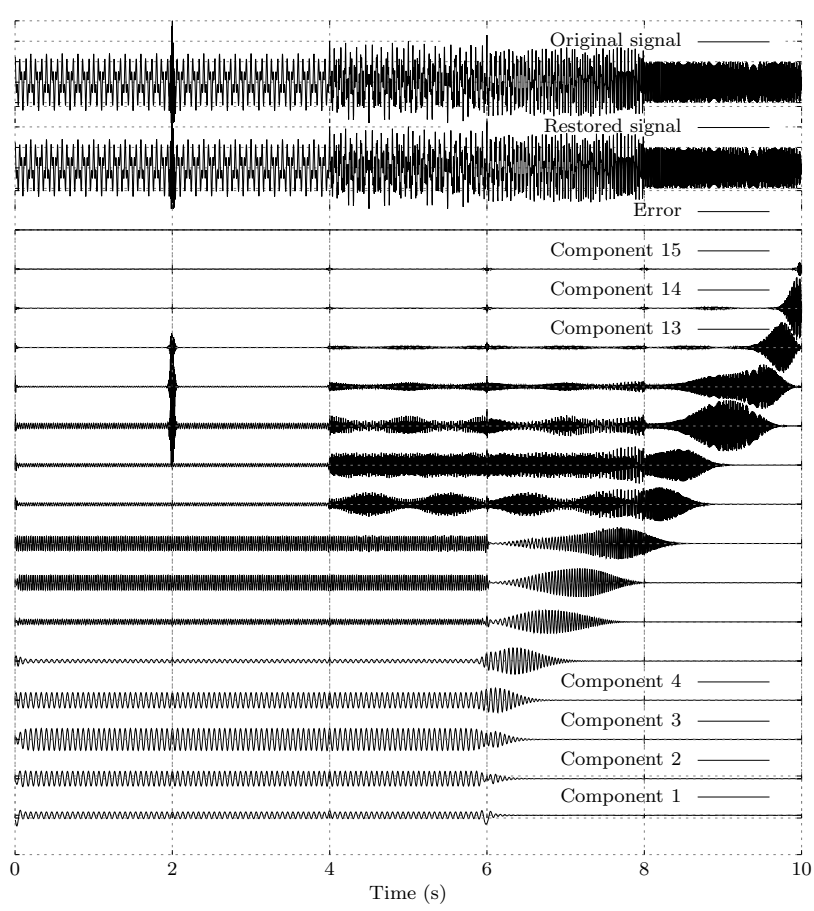

Figure 4: Synthetic signal decomposition using RI-SSA algorithm with $\mathrm{j}=200$ iterations, $\mathrm{K}=15$ components e $\mathrm{N}$ $=\mathrm{K}-\mathrm{K}+1$ recursions

In order to find the $s_{1}, \ldots, s_{5}$ components, Figure 1, Figure 5 presents the sum of similar components, from low to high frequency. It can be seen that the separation of the components with this method was better than that shown in Figure 2. Note that the sum of the components using the RI-SSA method Figure 5 is closer to the original components than the result obtained by means of SSA Figure 3.

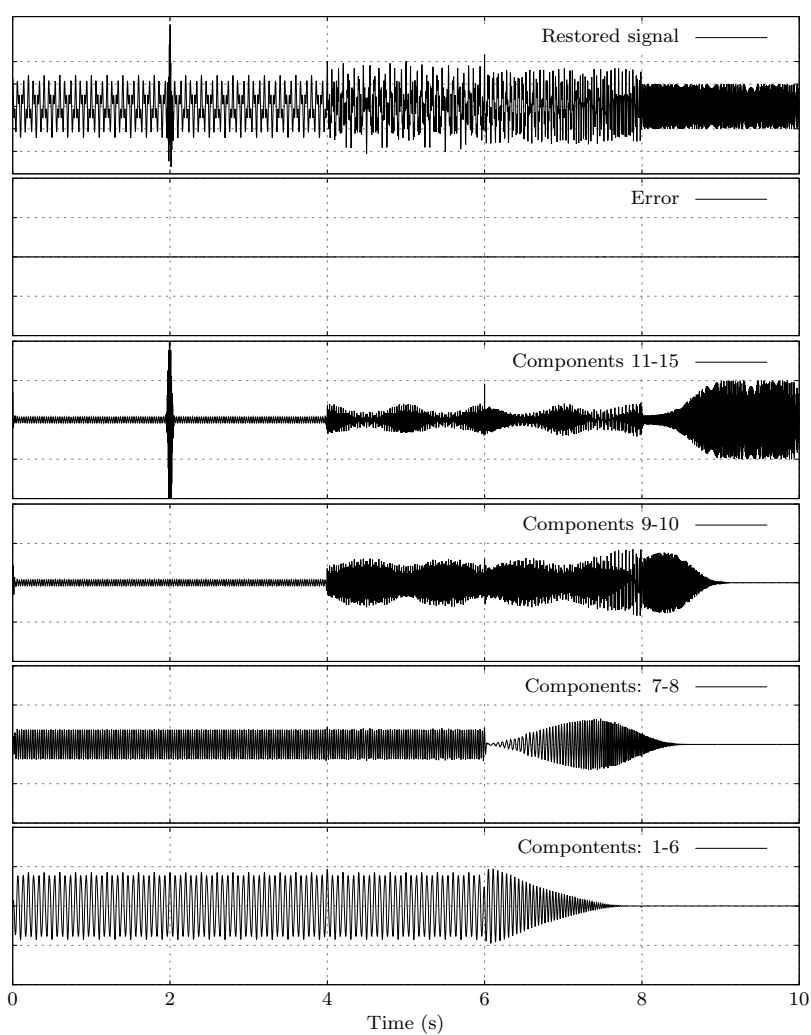

Figure 5: Signal components estimation with the sum of similar components

\section{Time-frequency Decomposition}

Signal analysis in the spectral domain is common in seismic processing. Using the Fourier transform (FT), a time series $s(t)$ passes to the frequency domain $S(f)$. The classical spectral representations are defined for stationary data and linear systems, these being the main characteristics of the limitations of such method. Over time, new methods have been developed aiming to overcome such limitations. Hence, the Fourier transform cannot be properly applied to signals whose frequency content varies significantly with time; FT is not suitable for non-stationary signals.

In the temporal representation $s(t)$, it is possible to know how the amplitude of the signal varies with time, casually providing information related to the frequency content of the signal. Considering the frequency domain representation $S(f)$, known as spectral analysis, it is possible to analyze different frequency components of the signal, as well as information related to the magnitude of each component.

In obtaining the joint information, it is necessary to directly represent the frequency content with the temporal characteristics. With this strategy, the $1-D$ signal is converted into a $2-D$ spectrum, where its dimensions are time and frequency, which means that, in this new domain, the complete spectrum shows the frequency content for each time. 


\section{Short-Time Fourier Transform}

In the short-time Fourier transform (STFT), it is assumed that the frequency varies a little in small time windows, the main problem being the temporal location. At finite time intervals the signal is approximately stationary. Normally the signal is divided into traces and the Fourier transform is calculated for each trace. Formally, by sliding the window $w(t-\tau)$ (equation 4), centered in instant $t_{n}$, through every seismic trace and applying the Fourier transform, it is possible to obtain the spectral variation with time. The mathematical expression for STFT is given by:

$$
\operatorname{STFT}(\tau, f)=\int_{-\infty}^{+\infty} s(t) w(t-\tau) e^{-2 i \pi f t} d t
$$

where $s(t)$ is the synthetic singal, $w(t-\tau)$ is the window, $\tau$ is the window center time, and $e^{-2 i \pi f t}$ is the Fourier kernel.

When you want to get a higher temporal resolution, the window $w$ has to be the smallest possible. On the other hand, large window will generate the opposite effect because it acts as a fine filter in the frequency domain, implying fine frequency sampling.

Figure 6 represents the original components decomposition using STFT, and Figure 7, after RI-SSA.

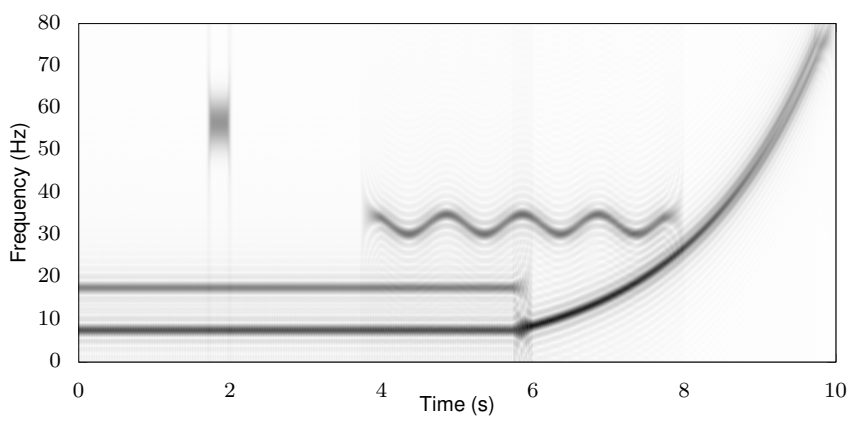

Figure 6: Time-frequency of the components (Figure 1) using STFT

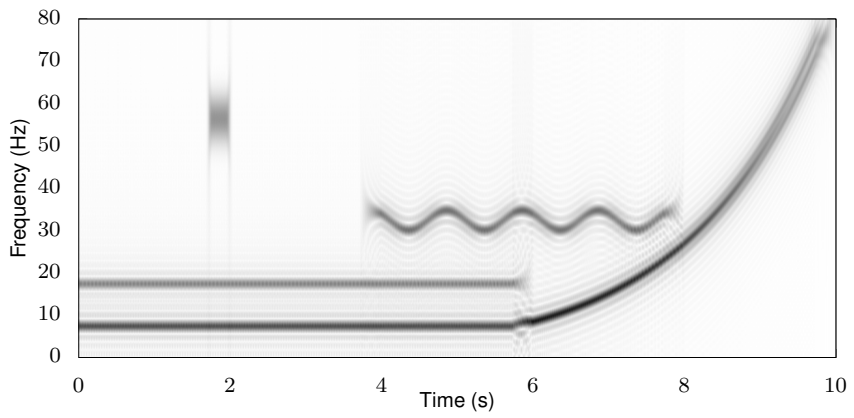

Figure 7: Time-frequency after RI-SSA (Figure 5) using STFT

\section{Wigner-Ville distribution of an analytic signal}

Para um sinal $z(t)$ amostrado uniformemente com intervalo $\Delta t$, a distribuição de Wigner-Ville discreta é dada por Boashash (1992):

For a uniformly sampled signal $z(t)$ with interval $\Delta \mathrm{t}$, the discrete Wigner-Ville distribution is given by Boashash (1992):

$$
W(t, f)=2 \sum_{k=0}^{N-1} z(t-k) z^{*}(t+k) e^{(-2 j \pi f k)}
$$

where the analytic signal $z(n)$ and its conjugate $z^{*}(n)$ corresponding to $x(n)$ given by:

$$
z(n)=x(n)+j H[x(n)]
$$

where $H[x(n)]$ represents the Hilbert transform of the signal $x(n), n=0, \ldots, N_{s}-1$, and $N_{s}$, the number of observations.

\section{Maximum Entropy Method}

Usually the power spectrum is estimated using the shortwindow Fourier transform (STFT) of the coefficients of the autocorrelation function $(F A C)$. However, the leakage effect "leakage", when the data is truncated, limits the Fourier transform. To obtain good resolution from a limited data series, (Burg, 1967) formulated the Maximum Entropy Method, constituting of a linear predictive filter, which gives the least quadratic error between the data and its predicted values. The basic form of the maximum entropy method is given by equation 7 :

$$
P(f)=\frac{E_{N_{c}} \Delta t}{\left|\sum_{n=0}^{N_{c}-1} c_{n} e^{-j 2 \pi f n \Delta t}\right|^{2}},
$$

where $P(f)$ is the power spectrum, $c_{n}, n=0, \ldots, N_{c}-$ $1, \quad\left(c_{0}=1\right)$ represent the prediction error operator (PEO) coefficients, also known as reflection coefficient and/or autoregressive parameter of order $N_{c}, E_{c}$ is the corresponding error energy, and $f$ is limited by the Nyquist interval $-1 /(2 \Delta t) \leqslant f \leqslant 1 /(2 \Delta t)$.

The power spectrum $P(t)$ is completely defined if the $c_{n}$ coefficients and the $E_{N_{c}}$ energy are known. The $P E O$ was estimated by the (Burg, 1967) algorithm directly from the analytic signal $z(n)$

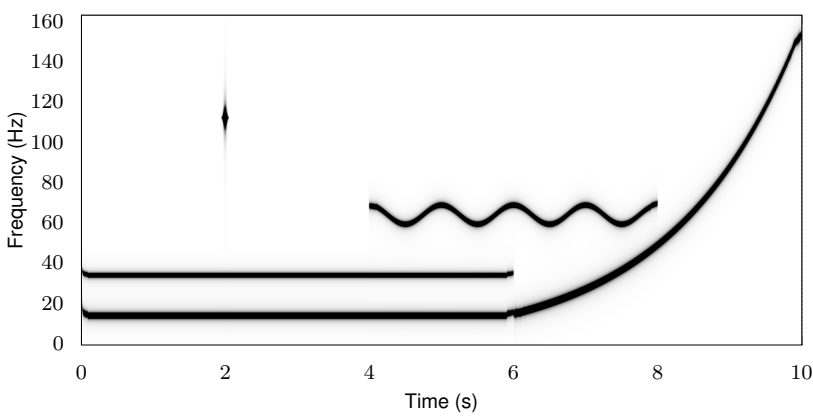

Figure 8: Time-frequency decomposition using WV-MEM

\section{Real data examples}

\section{Application of the SSA Algorithm in the Real Data}

As is known, by using SSA algorithm, it is possible from a seismic trace to find the corresponding eigenimages and/or eigentraces. Furthermore, it is known that each eigentrace corresponds to a certain frequency content. This technique 


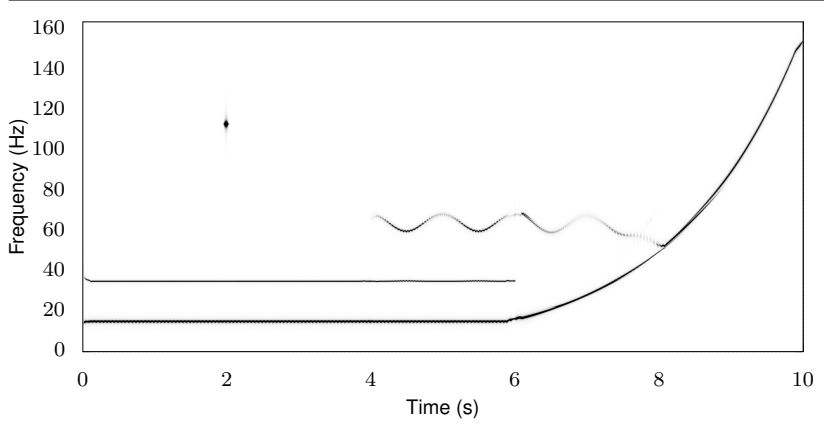

Figure 9: Decomposition using WV-MEM after RI-SSA

can be used to delete certain uninteresting parts of the data.

Figure 10(a) corresponds to a seismogram of the Recôncavo basin 3D seismic data (line Riacho São Pedro Jacuípe), Figure 10(b) represents the low frequency of the data, and Figure 10(c) represents the final result; note that the ground roll is still strongly present. Figure 11 represents the frequency spectrum of the real data, the low frequency containing the ground roll and the high frequency filtered data.

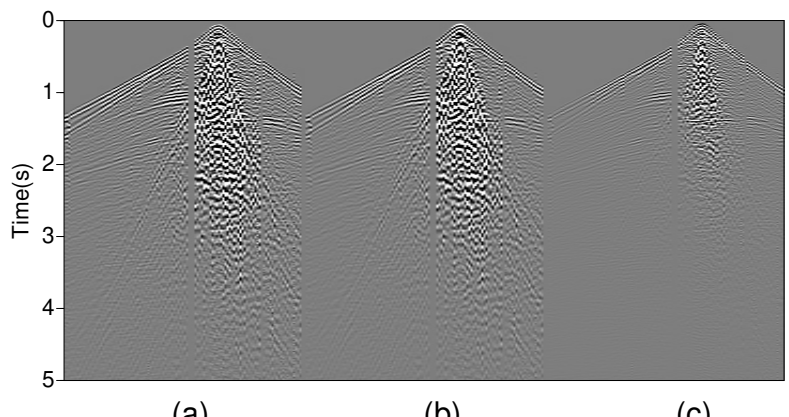

(a)

(b)

(c)

Figure 10: Seismograms of the real data in (a), low frequencies in (b), and final result in (c)

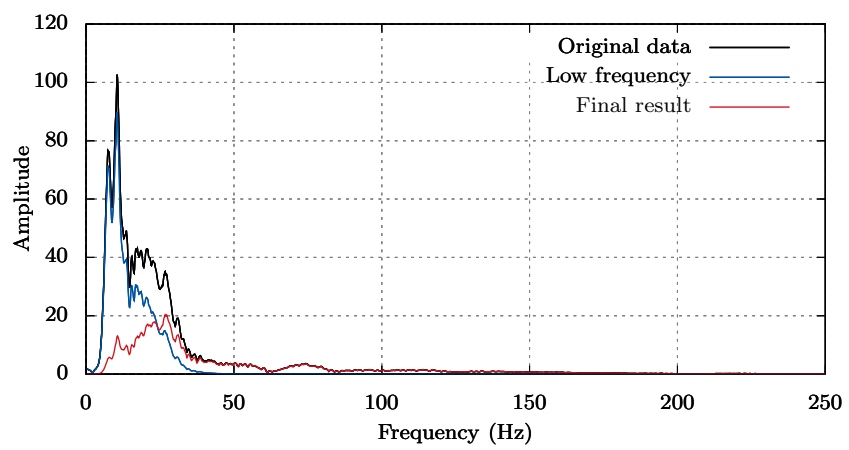

Figure 11: Data spectrum after SSA filtering

\section{Filtering ground roll using RI-SSA algorithm}

Note that the RI-SSA (Figure 4) was able to adequately separate the frequency components, and, applied to land data 12, this algorithm is capable of accurately filtering ground roll, increasing the signal-to-noise ratio.

From the frequency spectrum, Figure 13, it can be seen

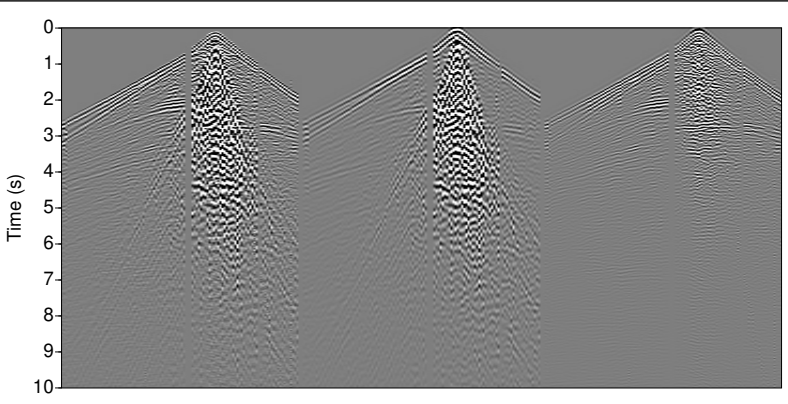

(a)

(b)

(c)

Figure 12: Seismograms of the real data in (a), low frequencies in (b), and final result in (c)

that the RI-SSA method was able to adequately separate the high and low frequency components, which can be used to totally remove the noise from the data.

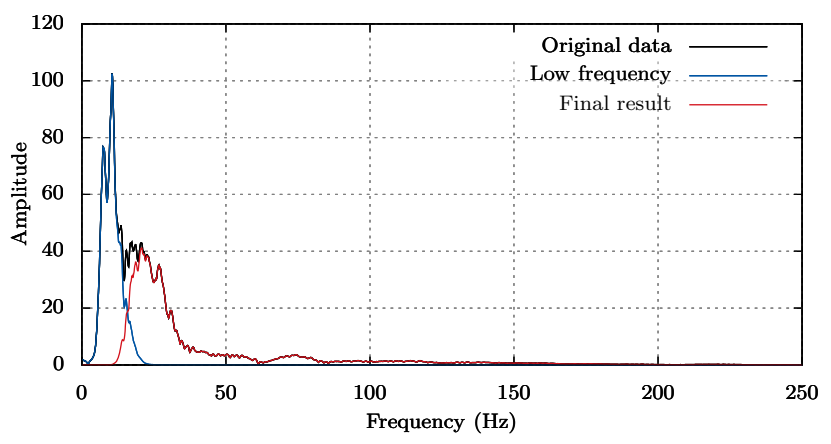

Figure 13: Data spectra after the use of RI-SSA

\section{Seismic attributes of real data}

Most of the extracted attributes were obtained using the WV-MEM spectrum in post-stacked data. As a geomorphological attribute, we have the instantaneous average frequency presented in Figure 15, extracted from Figure 14. The vertical resolution of the spectral section is observed due to the resolution of the WV-MEM method. Figure 16 refers to the variant attribute and corresponds to local deviation and frequency with respect to average frequency. And the instantaneous power as seen in Figure 17.

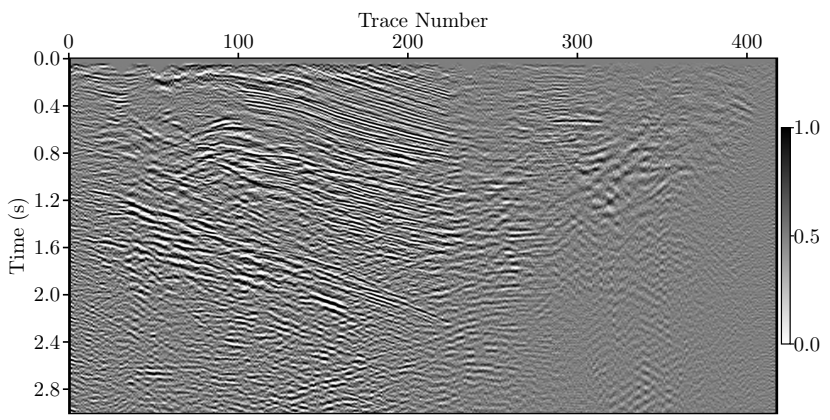

Figure 14: Original data 


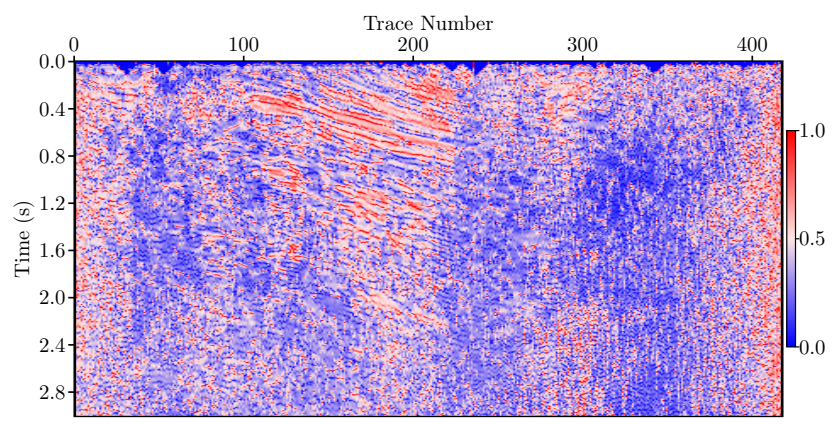

Figure 15: Average instantaneous frequency using WVMEM

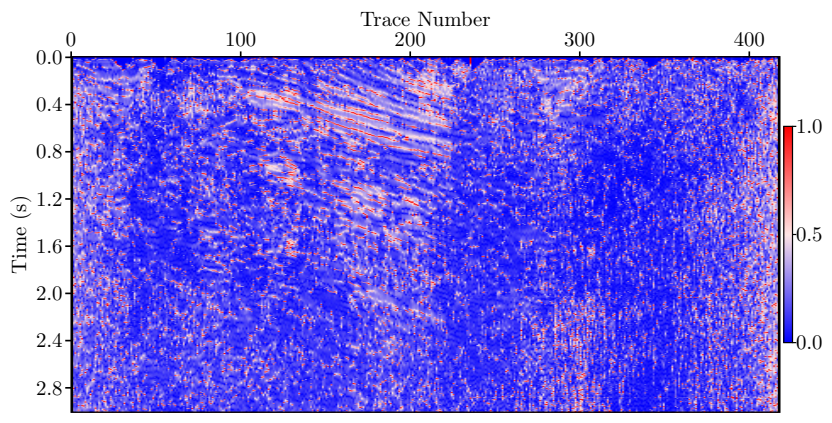

Figure 16: Average instantaneous variance using WVMEM

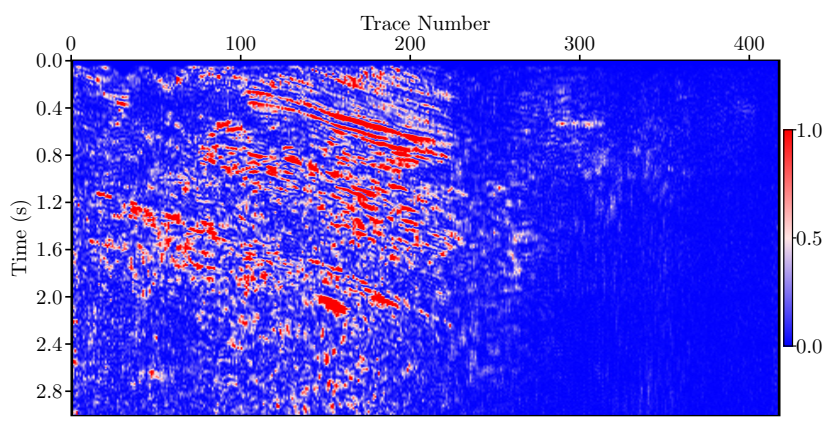

Figure 17: Instantaneous power

\section{Conclusion}

The RI-SSA method was able to decompose the signal into several intrinsic modes, as seen in Figure 4, with high separability. Recursions and iterations are essential for the separability of the components. This approach developed by Porsani et al. (2018) was used for the attenuation of ground roll, generating excellent results.

The Wigner-Ville maximum entropy distribution was obtained using the Burg method to extend the WignerVille kernel sequences using the prediction error operator, applying to each extended Kernel sequence the Fourier transform.

From the WV-MEM spectrum, it was possible to obtain the seismic attribute instantaneous average frequency and mean variance attributes with high resolution for the characterization of reservoirs.

\section{Acknowledgements}

This research was supported by CNPq and INCTGP/CNPq. The facility support from CPGG/UFBA is also acknowledged.

\section{References}

Andrews, H. C. and B. R. Hunt, 1977, Digital Image Restoration: Prentice-Hall.

Boashash, B., 1992, Estimating and interpreting the instantaneous frequency of a signal. I. Fundamentals: Proceedings of the IEEE, 80, 520-538.

Burg, J. P., 1967, Maximum entropy spectral analysis: Presented at the 37th Annual International SEG Meeting.

Freire, S. L. M., 1986, Applications of the singular value decomposition in seismic data processing: $\mathrm{PhD}$ thesis, Federal University of Bahia, Brazil [in portuguese].

Golub, G. H. and C. F. Van Loan, 2012, Matrix computations, volume 3: JHU Press.

Harris, T. and H. Yuan, 2010, Filtering and frequency interpretations of singular spectrum analysis: Physica D: Nonlinear Phenomena, 239, 1958-1967.

Porsani, M. J., M. G. Silva, and B. Ursin, 2017, Recursiveiterative zero-phase filtering via singular spectrum analysis: Presented at the 79th EAGE Conference and Exhibition 2017.

Porsani, M. J., B. Ursin, and M. G. Silva, 2018, Signal analysis and time-frequency representation using SSA and adaptive AR methods, in SEG Technical Program Expanded Abstracts 2018, 5022-5026, Society of Exploration Geophysicists.

Silva, M. G., 2015, Automatic stack using the local slope and new methods for seismic data processing in time domain: PhD thesis, Federal University of Bahia, Brazil [in portuguese].

Taner, M. T., J. S. Schuelke, R. OD́oherty, and B. E, 1994, Seismic attributes revisited, in SEG Technical Program Expanded Abstracts 1994, 1104-1106, Society of Exploration Geophysicists.

Tary, J. B., R. H. Herrera, J. Han, and M. van der Baan, 2014, Spectral estimation - What is new? What is next?: Reviews of Geophysics, 52, 723-749.

Zoukaneri, I. and M. J. Porsani, 2015, A combined WignerVille and maximum entropy method for high-resolution time-frequency analysis of seismic data: Geophysics, 80, O1-O11. 